\title{
Rapid onset of blindness due to meningeal carcinomatosis from an oesophageal adenocarcinoma
}

\author{
J.P. Teare, M. Whitehead, M.O. Rake and R.J. Coker ${ }^{1}$ \\ Departments of Medicine, Kent and Canterbury Hospital, Canterbury, Kent CT1 3NG; and ${ }^{1}$ Mount \\ Vernon Hospital, London, UK
}

\begin{abstract}
Summary: We report a 49 year old man who presented with left leg weakness, frontal headache, impaired concentration and dysphagia. He was thought to have a benign oesophageal stricture on barium swallow and oesophagoscopy though this was not initially biopsied. During admission his vision deteriorated so that he could only recognize bright light. Repeat oesophagoscopy demonstrated an oesophageal adenocarcinoma. The diagnosis of meningeal carcinomatosis was confirmed at post-mortem.

Sudden bilateral blindness is a common feature of meningeal carcinomatosis but, as this is rare, it is not commonly considered in the differential diagnosis of visual loss. The visual loss can be explained by vascular insufficiency in association with tumour cuffing of the subarachnoid space of the optic nerve sheath, by neuronal toxins, or other, as yet unknown, mechanisms.
\end{abstract}

\section{Introduction}

Meningeal carcinomatosis consists of diffuse or multifocal seeding of the leptomeninges and is a rare complication of metastatic cancer. It has protean clinical manifestations which often make diagnosis difficult. The most common features are headache, changes in mental state, cranial nerve palsies and neck stiffness. Visual failure as part of the syndrome of meningeal carcinomatosis was first noted in $1955^{1}$ and subsequently reported by $\mathrm{Katz}^{2}$ in 1961, where decreased vision was described in $44 \%$ of cases, though more recent reports ${ }^{3,6}$ suggest a less common association.

No patients with oesophageal adenocarcinoma have been described as developing meningeal carcinomatosis but the syndrome has been associated with adenocarcinoma of the stomach with a reported incidence of less than $5 \%$ of all cases reviewed..$^{3-7}$ We describe a patient who developed meningeal carcinomatosis secondary to a lower oesophageal adenocarcinoma.

\section{Case report}

A previously fit 49 year old, left handed man presented with a 6-month history of retrosternal

Correspondence: J.P. Teare, M.R.C.P., Gastrointestinal Laboratory, The Rayne Institute, St Thomas' Hospital, Lambeth Palace Road, London SE1 7EH, UK.

Accepted: 15 April 1991 burning pain, and recent dysphagia for solids. A barium meal showed a benign stricture at the lower end of the oesophagus. At oesophagoscopy, the stricture was easily dilated to 54 French.

He re-presented 4 months later with 3 weeks of frontal headache, reduced libido, impaired concentration, followed by 2 weeks of increasing left leg weakness. For the week prior to admission he had developed a fluctuating level of consciousness culminating in an episode of loss of consciousness associated with urinary incontinence and limb jerking. On examination there was blurring of the disc margins on the right, Grade four weakness of left leg hip flexion, disorientation in time and no recall for an address. Visual acuity was N18 in both eyes with full visual fields and normal pupillary reflexes.

Full blood count, urea and electrolytes, liver function tests, blood glucose and chest radiograph were all normal. Cranial computed axial tomography (CT) showed no abnormality. An electroencephalogram (EEG) was normal, cerebrospinal fluid (CSF) examination showed 3 lymphocytes per $\mathrm{ml}$, no red cells, protein concentrations of $0.09 \mathrm{~g} / \mathrm{l}$ and glucose of $4.0 \mathrm{mmol} / 1$. Culture and serology were normal. Borrelia serology, VDRL and blood cultures were negative. Auto-antibodies screen and C-reactive protein were normal.

His conscious level improved and he became fully orientated. He was discharged home taking phenytoin $100 \mathrm{mg}$ three times daily. However, over the next month, his frontal headaches returned, he 
became clumsy with his left hand, and he noticed some dimming of his vision. He again complained of dysphagia to solids. On re-examination there was left finger-nose and heel-shin ataxia with normal power. His neuro-ophthalmology was normal. Over the next 4 days he experienced intermittent paralysis. During episodes his pupils dilated widely, plantar responses became extensor and he was semiconscious, responding sluggishly to verbal commands.

Repeat endoscopy showed an adenocarcinoma of the lower third of the oesophagus. A CT scan of the thorax demonstrated the tumour extending for $14 \mathrm{~cm}$ locally with no other spread. A repeat CT scan of the head was normal. An EEG at that time demonstrated a generalized abnormality with irregular theta activity and a diffuse excess of slow activity.

His vision deteriorated until he could only recognize bright lights, and no figures. On examination the pupils were dilated at $6 \mathrm{~mm}$, the right eye reacted directly and consensually, whereas the left eye did not. There were no afferent pupillary defects. Fundoscopy was normal. There were no other abnormal cranial nerve signs. Over the next 2 days he developed corneal oedema before dying from cardiopulmonary arrest.

\section{Pathological findings}

General autopsy and cerebral examination, were performed. The visual pathways were given special attention. An extensive adenocarcinoma was found in the lower part of the oesophagus and lesser curvature of the stomach. The liver and lymph nodes were free of disease. The brain, meninges and optic pathways were not grossly involved. Around the midbrain the meninges appeared slightly opaque. Blocks taken from the frontal lobe, temporal lobe, cerebellum, pons and medulla, showed patchy leptomeningeal deposits of cells from a metastatic adenocarcinoma (Figure 1). The optic nerve sections were examined under light microscopy. The leptomeninges contained malignant cells with large hyperchromatic nuclei and abundant cytoplasm. Similar cells were seen in the leptomeninges lifted from the optic chiasm, where cells could be seen surrounding a leptomeningeal vessel (Figure 2).

\section{Discussion}

Blindness is a common feature of meningeal carcinomatosis but the latter is rare and so not commonly considered in the differential diagnosis of visual loss. This patient was typical in that he developed blindness simultaneously in both eyes. ${ }^{8}$

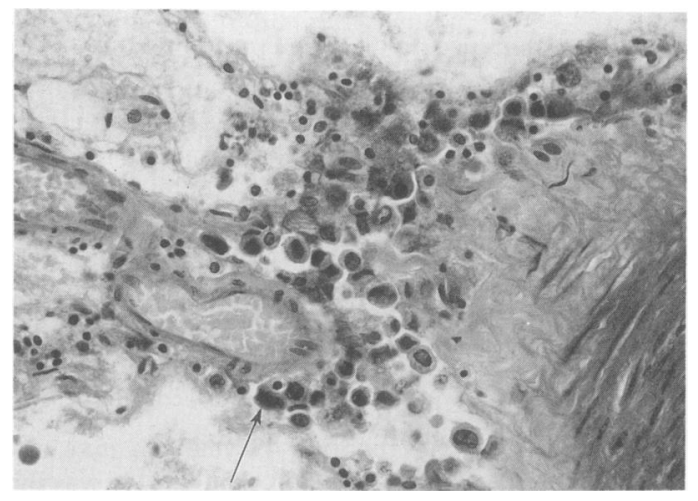

Figure 1 This photomicrograph shows leptomeninges of the cerebellum infiltrated by cells with hyperchromatic nuclei and abundant eosinophilic cytoplasm (arrow) characteristic of an adenocarcinoma. Haematoxylin and $\operatorname{eosin} \times 140$.

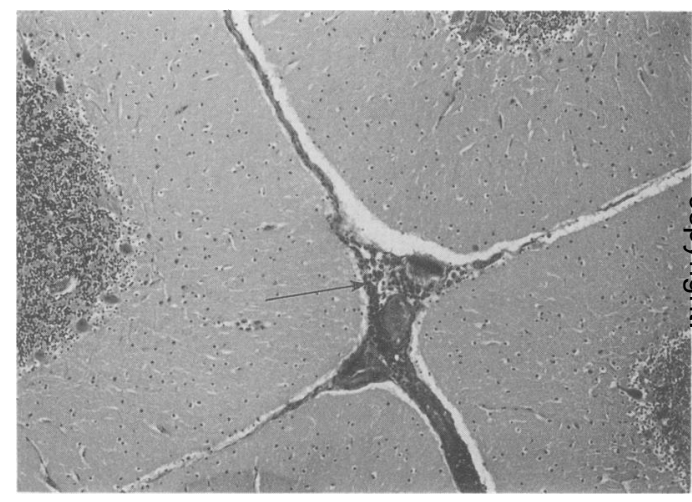

Figure 2 This photomicrograph shows large pleomorphic cells with hyperchromatic nuclei infiltrating the meninges of the optic chiasm and surrounding the large leptomeningeal vessel. Haematoxylin and eosin $\times 140$.

Meningeal carcinomatosis could have been suggested in this patient by the combination of headache, blindness and spinal root involvement. Other recognized features include meningeal irritation, cranial nerve involvement, areflexia and dementia. Lumbar puncture findings may be characteristic with a reduced glucose, increased protein and the presence of malignant cells detected by millipore-filter examination. In this patient, however, the lumbar puncture was normal, although cytology was not performed.

The mechanism of dissemination of tumour to the leptomeninges is not clear. Some sources suggest direct invasion of the cerebrospinal fluid through the choroid plexus ${ }^{1}$ or through leptomeningeal vessels; ${ }^{9}$ others suggest secondary invasion from tumour in parenchyma or nerve roots. ${ }^{10}$ It is 
likely, however, that the metastasis is blood borne, as the choroid plexus is frequently involved. Grain and $\mathrm{Karr}^{11}$ also suggest retrograde dissemination of tumour along perineural lymphatics and sheaths. In keeping with most published series, ${ }^{3-6}$ where adenocarcinoma is the most frequent histological type of carcinoma to metastasize to the leptomeninges, our patient had an adenocarcinoma confined to the oesophagus, though presumably of gastric origin.

The exact pathophysiology of visual loss is not clear. Histological examination generally shows marked tumour-cuffing of the subarachnoid space of the optic nerve sheath with sheets of metastatic cells. Susac ${ }^{8}$ suggested a vascular basis for the blindness in association with cuffing, though others ${ }^{1}$ could find no evidence for vascular inter- ference. Direct invasion of the nerve itself is very unusual though Altrocchi ${ }^{12,13}$ points out that the intraorbital portion of the nerve is rarely examined at post-mortem. In this patient the leptomeningeal vessels can be seen surrounded by malignant cells in many of the optic nerve sections. Other hypotheses include neuronal toxins ${ }^{1,14}$ and chronic papilloedema, found to be present in a proportion of cases, but not in this case. Some cases, however, remain inadequately explained by any of these mechanisms.

Metastatic carcinomatosis causing blindness is a diagnosis commonly missed when not considered in patients with bizarre neurological presentations. Once considered, however, the diagnosis can often be confirmed by lumbar puncture. ${ }^{15-17}$

\section{References}

1. Fischer-Williams, M., Bosanquet, F.D. \& Daniel, P.M Carcinomatosis of the meninges. A report of three cases. Brain 1955, 78: 42-58.

2. Katz, J.L., Valsamis, M.O. \& Jampel, R.S. Ocular signs in diffuse carcinomatous meningitis. Am J Ophthalmol 1961, 52: 681-690.

3. Olson, M.E., Chernik, N.L. \& Posner, J.B. Infiltration of the leptomeninges by systemic cancer. Arch Neurol 1974, 30: 122-137.

4. Wasserstrom, W.R., Glass, J.P. \& Posner, J.B. Diagnosis and treatment of leptomeningeal metastases from solid tumours. Cancer 1982, 49: 759-772.

5. Gonzalez-Vitale, J.C. \& Garcia-Bunuel, R. Meningeal carcinomatosis. Cancer 1976, 37: 2906-2911.

6. Little, J.R., Dale, A.J. \& Okazaki, H. Meningeal carcinomatosis. Arch Neurol 1974, 30: 138-143.

7. Theodore, W.H. \& Gendelman, S. Meningeal carcinomatosis. Arch Neurol 1981, 38: 696-699.

8. Susac, J.O., Lawton Smith, J. \& Powell, J.O. Carcinomatous optic neuropathy. Am J Ophthalmol 1973, 76: 672-679.

9. Price, R.A. \& Johnson, W.W. The central nervous system in childhood leukaemia. 1. The Arachnoid. Cancer 1973, 31: 520-533.
10. Griffin, J.W., Thompson, R.W., Mitchinson, M.J. et al. Lymphomatous leptomeningitis. Am J Med 1971, 51: 200-208.

11. Grain, G.O. \& Karr, J.P. Diffuse leptomeningeal carcinomatosis. Clinical and pathologic characteristics. Neurology 1955, 5: 706-722.

12. Altrocchi, P.A., Reinhardt, P.H. \& Eckman, P.B. Blindness and meningeal carcinomatosis. Arch Ophthalmol 1972, 88: $508-512$.

13. Altrocchi, P.H. \& Eckman, P.B. Meningeal carcinomatosis and blindness. J Neurol Neurosurg Psychiatr 1973, 36: 206-210.

14. Appen, R.E., De Venecia, G., Selliken, J.H. \& Giles, L.T. Meningeal carcinomatosis with blindness. Am J Ophthalmol 1978, 86: 661-665.

15. Berg, L. Hypoglycorrhachia of non-infectious origin: diffuse meningeal neoplasia. Neurology 1953, 3: 811-824.

16. Fishman, R.A. Studies of the transport of sugars between blood and cerebrospinal fluid in normal states and in meningeal carcinomatosis. Trans Am Neurol Assoc 1963, 88: $114-118$.

17. Kim, Y.S. \& Resnick, J.S. Hypoglycorrhachia with meningeal carcinomatosis. Ann Intern Med 1965, 63: 115-121. 